Introduction Despite the global strides made in palliative and hospice care, organisational challenges continue to negatively impact the accessibility to care and adequacy of pain and symptom management for patients and their family members. Nurses play a fundamental role in advocating for and providing high-quality palliative and end of life care, however, there is limited understanding of their occupational wellbeing. The purpose of this presentation is to explore the occupational resources and demands in palliative nursing practice through the development and psychometric evaluation of the Palliative Care Nursing-Job Resources (PCN-JR) Scale and Palliative Care Nursing- Job Demands (PCN-JD) Scale.

Methods This study used a three-phase process of instrument development and psychometric evaluation within a provincewide cross-sectional design. Phase 1 involved a thematic analysis of qualitative data, palliative expert consultation, and a content validation index in the development of a 64-item PCN-JR Scale and 72-item PCN-JD Scale. Phase 2 consisted of a pilot survey of 55 nurses and use of item discrimination analysis to estimate internal consistency reliability and reduce the length of each scale. Exploratory factor analysis was used in Phase 3 to further test the modified scales in a provincewide survey of $n=377$ nurses who provide palliative and end of life care.

Result Exploratory factor analysis of the 32 items related to palliative job resources favoured an 8 -factor structure, accounting for $62 \%$ of the variance, Cronbach's alpha 0.90 . The 36 items related to palliative job demands favoured an 8 -factor structure, accounting for $61 \%$ of the variance, Cronbach's alpha 0.93 .

Discussion The Palliative Care Nursing-Job Resources (PCNJR) Scale and Palliative Care Nursing- Job Demands (PCN-JD) Scale are valid and reliable, and have broad applicability to better understand the occupational wellbeing of palliative care nurses. Further research is necessary to further evaluate their psychometric properties from a national and international perspective.

\section{CAN COPING STRATEGIES ALLEVIATE THE LINK BETWEEN WORKPLACE AGGRESSION AND BURNOUT? THE ROLE OF SEEKING INSTRUMENTAL OR EMOTIONAL SOCAL SUPPORT}

\begin{abstract}
${ }^{1}$ Ellen Delvaux*, ${ }^{1,2}$ Whitney Van den Brande, ${ }^{1}$ Martijn Schouteden, ${ }^{1}$ Maarten Sercu, ${ }^{2}$ Elfi Baillien, ${ }^{2}$ Anja Van den Broeck, ${ }^{1,3}$ Lode Godderis. ${ }^{1}$ IDEWE, External Service for Prevention and Protection at Work, Leuven, Belgium; ${ }^{2} \mathrm{KU}$ Leuven, Work and Organisation Studies, Brussel, Belgium; ${ }^{3} \mathrm{KU}$ Leuven, Centre for Environment and Health, Leuven, Belgium
\end{abstract}

\subsection{6/oemed-2018-ICOHabstracts. 1616}

Introduction Aggression at the workplace occurs frequently. Workplace aggression negatively impacts employees' psychological well-being. In the current research, we investigated whether coping strategies alleviated the negative impact of workplace aggression on one important indicator of employees' psychological well-being, namely burnout (risk). More specifically, we examined whether the coping strategies seeking instrumental or emotional social support moderated the relationship between workplace aggression and burnout symptoms.
Methods Questionnaires were administered to employees of a large Belgian hospital. In total, 1544 employees participated (response rate $=44 \%$ ). The scale measuring workplace aggression consisted of three items $(\alpha=0,79)$. Burnout risk was measured with the two most central subscales of the Dutch version of the Maslach Burnout Inventory (UBOS), namely emotional exhaustion (5 items; $\alpha=0,92$ ) and depersonalization (4 items; $\alpha=0,86$ ). Finally, from the COPE questionnaire, we used the subscales seeking instrumental social support ( 4 items; $\alpha=0,92)$ and emotional social support ( 4 items; $\alpha=0,92$ ). We tested our hypotheses using hierarchical regression analyses.

Result Replicating previous research, we found that workplace aggression increases both the levels of emotional exhaustion and depersonalization. Furthermore, we found that seeking instrumental social support is a better coping strategy than seeking emotional social support: the former decreases burnout symptoms, whereas the latter may even increase burnout symptoms. Finally, we found that the coping strategy seeking instrumental social support alleviated the relationship between workplace aggression on the one hand and emotional exhaustion and depersonalization on the other. Seeking emotional social support only moderated the relationship between workplace aggression and depersonalization: the level of depersonalization increased for employees with lower scores on this coping strategy when they encountered workplace aggression; employees scoring high on this coping strategy had increased levels of depersonalization regardless of experienced workplace aggression.

Discussion Our findings will be discussed in light of its theoretical and practical contributions.

\section{ALL EMPLOYEES RESILIENT THROUGH AGGRESSION AFTERCARE': THE DEVELOPMENT OF A DIGITAL PLATFORM SUPPORTING AGGRESSION AFTERCARE IN HEALTH CARE TEAMS}

${ }^{1}$ E Delvaux* ${ }^{2} \mathrm{H}$ Leirs, ${ }^{2} \mathrm{~J}$ Schrijvers, ${ }^{3}$ Konovaloff, ${ }^{1,4} \mathrm{~L}$ Godderis. ${ }^{1}$ IDEWE, External Service for Prevention and Protection at Work, Leuven, Belgium; ${ }^{2}$ UCLL, University Colleges LeuvenLimburg, Diepenbeek, Belgium; ${ }^{3}$ ICOBA, Knowledge and Expertise Centre on Aggression, Brussels, Belgium; ${ }^{4}$ KU Leuven, Centre for Environment and Health, Leuven, Belgium

\subsection{6/oemed-2018-ICOHabstracts. 1617}

Introduction Health care workers are often confronted with aggression, impairing their well-being at work. Aggression aftercare, adjusted to the personal needs of victims, may facilitate their recovery, return to and well-being at work.

Methods We used three methodologies to identify the most important needs for employees confronted with workplace aggression. First, fictive aggression incidents were reenacted in four organisations. After each scene, actors and audience discussed about impact and needs after encountering such incidents. Second, aggression experts $(n=10)$ were interviewed about existing and ideal aggression procedures within their institutions. Third, a diary questionnaire (of two weeks) was administered to employees $(n=11)$ that recently encountered workplace aggression.

Results Bringing together the input from the different methodologies by defining common and frequently re-occurring 
themes, we identified the most central needs of aggression victims: the need for acknowledgement, the need for support by colleagues and supervisor and the need for openness about aggression. Based on these needs, we conceptualised a program to facilitate aggression aftercare in teams. More specifically, on a digital platform, aggression victims can report that they encountered aggression, what impact this has on them and which colleague should contact them to talk about it. After saving the incident on the platform, an e-mail cascade will be set in motion to notify different actors about their responsibilities in the process of providing aftercare. The 'aftercare'-colleague will be most central to the aftercare process, supporting the victim both emotionally and practically. Depending on the rated impact, the supervisor will also play a central role in the aftercare process.

Conclusion Because aggression victims can personalise their incident report (by choosing the incident's impact and their preferred 'aftercare'-colleague), the aftercare will be more adjusted to their needs, which may increase resilience after aggression. The program effectiveness will be tested within a health care setting.

\section{VIOLENCE AGAINST INTERNS AT IBN ROCHD UNIVERSITY HOSPITAL OF CASABLANCA, MOROCCO}

I El Amri*, S Touil, B Benali, A El Kholti. Casablanca Faculty of Medicine and Pharmacy, Hassan II University, Morocco

\subsection{6/oemed-2018-ICOHabstracts. 1618}

Introduction Workplace violence in health care settings is a significant problem world wide. The aim of this study is to identify the proportion of interns affected by all three types of violence, the study of it's sources and consequences.

Methods This descriptive study was conducted between April and May 2017. We've tried to collect data from all the 100 interns that work in the university hospital of Casablanca, but only managed to get information from 60 of them. The study was done by the help of a questionnaire that included questions about sociodemographic characteristics, physical violence, verbal violence, sexual harassment and working conditions.

Results Participants were between 21 and 26 years of age. $67,8 \%$ were female. history of physical violence was found in $23,3 \%$ of the cases, the aggressor was either the patient or his family in $85,7 \%$ of the times. We found that $88,3 \%$ of the interns experienced episodes of verbal abuse, at least once every three months in $34,6 \%$ of the cases. Sexual abuse was seen in $11,7 \%$ of the cases, where $71,5 \%$ of the victims were female. Of the participants, only $58,3 \%$ said that they were encouraged to report those types of incidents.

Discussion The most frequent type of violence amongst interns was verbal in nature. Previous studies showed the same results (that verbal abuse occurs more often than physical or sexual harassment). The study shows also that patients' companions and patients themselves do the most violence, which confirms what other studies found. Sexual harassment rate was found to be $62,5 \%$ in women in a study in Turkey which confirms what we found.

Conclusion The results of the study suggest that violence is a major problem among interns and serious measures need to be taken immediately to avoid the growing number of these incidents.

\section{STRESS AMONGST ELEMENTARY AND HIGH SCHOOL TEACHERS}

B Benali*, I El Amri, F El Youssefi, A El Kholti. Casablanca Faculty of Medicine and Pharmacy, Hassan II University, Morocco

\subsection{6/oemed-2018-ICOHabstracts. 1619}

Introduction Teachers, as in other professions, are exposed to different types of problems within the field of occupational medicine. Generally, teachers heavily stressed show signs of psychological distress that usually shows as severe anxiety, low psychological comfort and low job satisfaction.

Methods The questionnaire was distributed within elementary, middle and high schools in the district of Hay Hassani, Casablanca. All subjects voluntarily accepted to be on the study. We've distributed 300 questionnaires but only gathered 140 .

Results From 140 subjects, 58,06\% were female, 26\% worked in primary schools, $51 \%$ in middle schools and $47 \%$ in high schools. Almost half of them $(48.38 \%)$ have been teaching for over 20 years. Of the participants, $31,14 \%$ never felled energetic, full of life or being eager to go through every new day. The auto evaluation of mental health found that 20,16\% rated their mental health as being medium to poor. Burnout was felt by $36,51 \%$ of the respondents once or more than once per month and $26,63 \%$ say that they always have a heavy workload. Roughly half of the participants (44\%) were not satisfied with their jobs.

Discussion In a Suiss study, they found that more than $80 \%$ of teachers showed medium burnout signs. In our study $37 \%$ showed exhaustion signs once or more every month which indicates that there is a disproportion between individual possibilities and the reality of working conditions. In a survey conducted by the autonomous federation of education of Quebec, they found that a heavy workload is the main determinant of stress experienced by teachers which explains our findings.

Conclusion Professional stress affects the well being of teachers. The main prevention mechanism that can be used is self management which represents the process that teachers have the most control on.

\section{EMPLOY MENTAL HEALTH AND EMOTIONAL INTELLIGENCE: STUDY OF TEACHERS WORKING IN PRIVATE AND GOVERNMENT SECTORS}

Indu Rathee. Associate Professor, Tika Ram College Of Education, Sonipat, Haryana(India)

\subsection{6/oemed-2018-ICOHabstracts. 1620}

Introduction Teacher's mental health is influenced by various factors like working conditions, professional development opportunities, gender, age, work experience, work load etc. With the changing socio- economic scenario, the values of teacher and their professional concern with the job have forcibly undergone a change, which adversely affects the mental health of the teacher. Being emotionally intelligent is not only crucial, but a necessary condition for a successful life. This research article describes the mental health and emotional intelligence of school teachers working in private and government institutions with regard to gender, age and the working conditions in which they work.

Method The researcher used the descriptive survey method for the present study. For data collection, the investigator used the 'Employee's Mental Health Inventory' developed by 